\title{
Enhancing the Tablet Images using Noise Reduction Algorithms by Analyzing Different Color Models
}

\section{A.B. Dhivya, M. Sundaresan}

\begin{abstract}
Unidentified tablets are challenges to both patients and healthcare professionals. Using these unknown tablets results in undesirable reaction of drug and also it is foundation to ill health that leads to death even sometimes. Thus, recognition of unidentified tablets is a significant task in medical industry. Identification of tablets is one of the major concerns for public and pharmacists, which can be carried out by means of either text-based or image-based methods. The tablet identification system is focused on removing noise from the tablet images using algorithms like Independent Component Analysis (ICA) and Discrete Wavelet Packet Transmission (DWPT). The three color space models, i.e., RGB (Red-Green-Blue), YCbCr (Y-Luma, CChroma of blue and red components) and HSV (Hue-SaturationValue) are examined for their efficiency on removing noise from tablets. For each color space model, the two denoising algorithms, ICA and DWPT are analyzed and applied. The result is interpreted using metrics like PSNR, FoM, MSSI and Speed. Experimental results proved that denoising with HSV color space model gives maximum efficiency when used with ICA and DWPT-based tablet identification systems.
\end{abstract}

Keywords: Color Space Model, Tablet Retrieval, Denoising, Wavelet Packets, ICA, DWPT, Reference Image, Consumer Image.

\section{INTRODUCTION}

Combination of algorithms in information technology with healthcare is a popular strategy used, to develop systems that can help both physicians and patients, in order to solve health problems efficiently and effectively. These systems focus on different applications of healthcare. Some examples of such applications include e-scripting, e-disease information, e-pharmacopoeia, and e-tablet identification [1]. e-medical illustration has taken out information from query tablets to regain similar tablets from tablet image database. These systems help people to recognize pills either by using external features (like name, manufacturer details, disease symptoms) or by using physical or appearance features (like size, color or shape). This structure aims to reduce instability during treatment and increase patient confidence in healthcare system [2]. One of the major issues faced by this system is the lack of techniques that can deliver the visual quality and size

\section{Revised Manuscript Received on December 30, 2019.}

* Correspondence Author

A.B. Dhivya*, Department of Information Technology, Bharathiar University, Coimbatore, Tamilnadu, India

M. Sundaresan, Department of Information Technology, Bharathiar University, Coimbatore, Tamilnadu, India

(C) The Authors. Published by Blue Eyes Intelligence Engineering and Sciences Publication (BEIESP). This is an open access article under the CC BY-NC-ND license (http://creativecommons.org/licenses/by-nc-nd/4.0/) standardizations required to perform tablet identification meaningful. These two steps are considered as preprocessing algorithms during tablet identification and retrieval. Tablet image size variations problem can be successfully handled using image interpolation techniques and the previous paper [3] described various interpolation techniques that can be used to produce standard size tablet images. This paper showed that the usage of image standardization techniques which improved the accuracy of tablet identification.

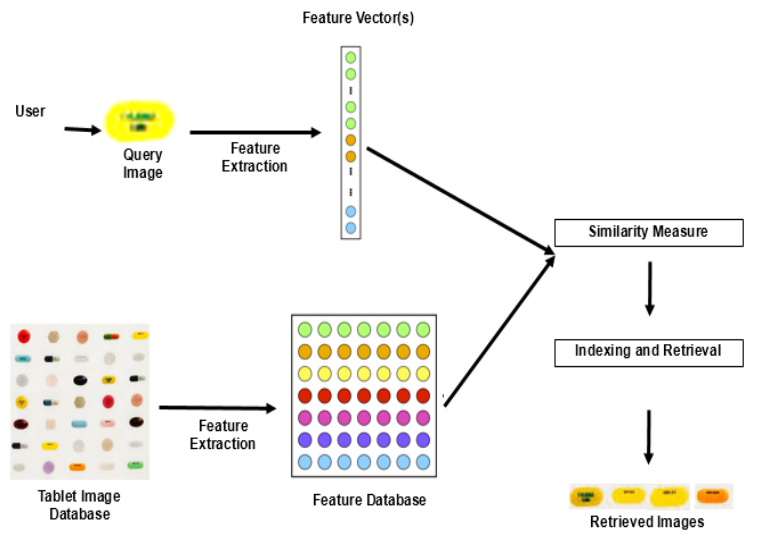

Fig. 1. Block Diagram of Image Query based Tablet Identification System

This paper is concerned with the removal of noise present in the tablet images, so as to improve its visual quality. Presence of noise in tablet image degrades the performance of tablet identification and hence is considered as an important step. Two noise removal algorithms based on Independent Component Analysis (ICA) and Discrete Wavelet Packet Transformation (DWPT) are analyzed and compared. The paper also analyzes a variety of color space models that can effectively assist the noise removal algorithm. In this paper, three frequently used color space models are described and compared. They are RGB (RedGreen-Blue), YCbCr (Y-Luma, C-Chroma of blue and red components) and HSV (Hue-Saturation-Value). The enduring part of the paper is structured as follows. Section 2 presents the review of literature. Section 3 presents the working of the Wavelet Packet Transmission-based Denoising Algorithm (WPTDA) and Independent Component Analysis-based Denoising Algorithm (ICADA). Section 4 presents a brief description on the three selected colour space models.

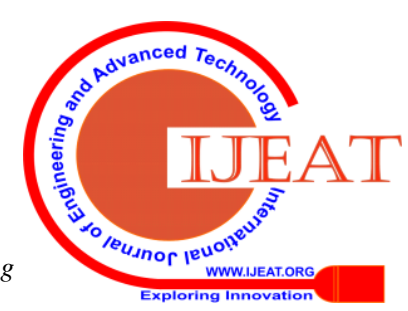


Section 5 presents the results of performance analysis conducted to identify the best method for denoising tablet image. Section 6 summarizes the work with future research directions.

\section{REVIEW OF LITERATURE}

Nalini, P. and Malleswari, B.L. (2016) [4] presented a color histogram using color space methods like RGB, HSV, YCbCr, CIE-LAB and CIE-XYZ. Several queries like people, animals, Food and outdoor scenes were used to compare the performance of content based image retrieval system. To measure the performance of the proposed system, the parameters precision, MAP and recall are used. The COREL database is used for retrieval purpose and the color features extracted from HSV color model performed better when compared to other color space models.

Mirarab, M.R., Dehghani, H. and Pourmohammad, A. (2010) [5] proposed a novel based wavelet based ICA technique using Kurtosis for blind audio source separation. To solve this problem, this paper used the independent component analysis which seperates the sources in the mixtures. The observations are made using wavelet packets decomposition and Kurtosis criterion.

Joblove, George H., and Donald Greenberg (1978) [6] proposed the way for representing image in HSV color space in which Hue denotes the purest form of color, Saturation contains the color with less white component and Value represents the intensity of color. This HSV is represented in cylindrical coordinate systems.

\section{DENOISING ALGORITHMS}

Noise is ubiquitous in almost all acoustic environments. Presence of noise in tablet images degrades spatial and contrast resolution and obscures the underlying structure of the image and therefore has a negative impact (that is, reduced accuracy) during identification. Due to these reasons, noise removal is a vital part of automatic tablet identification system. In this paper, to remove Gaussian noise present in tablet images, two algorithms, namely, WPTDA and ICADA, are proposed and are described in the following sections.

\section{A. Discrete Wavelet Packet Transmission based Denoising Algorithm}

Several studies have used Discrete Wavelet Transformation (DWT) for denoising images and speech signals [7]. Inspite of its general success, DWT fails to capture high frequency information accurately, especially at lower bit rates where such information is lost as quantization noise [8]. One way of solving this problem is to use Discrete Wavelet Packet Transformation (DWPT). The DWPT-based noise removal algorithm proposed in this paper consists of the steps shown in Fig. 2.

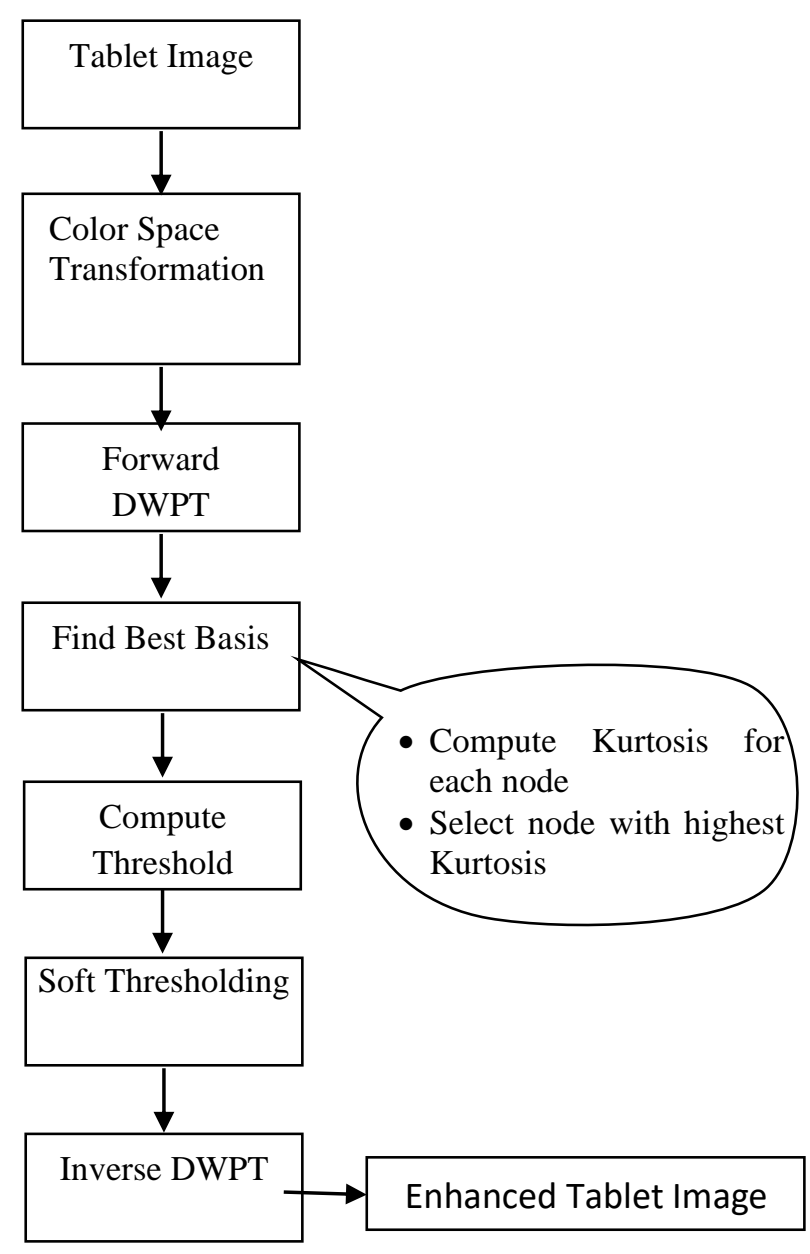

Fig. 2. Steps in WPTDA

DWPT has several good localization properties that provides advantages with both time and frequency domains. These properties aid in characterizing the structure and texture of images at various levels of resolutions and also improves edge detection task. Thus, DWPT-based noise removal algorithm can remove unwanted pixels (noise) while preserving edge details.

The algorithm begins with the application of wavelet packet decomposition to the two-dimensional tablet image. This results with a set of wavelet packet coefficients, which are represented as a wavelet packet tree where left branch represents low pass filtering, right branch represents high pass filtering and the middle branches represents the pass horizontal / high pass vertical filtering and the high pass horizontal / low pass vertical filtering, respectively. The wavelet packet coefficients of tablet image and noise have different properties at different scale. This fact is exploited during noise reduction/removal. By constructing an evaluation criterion, the WPT, using mathematical methods can process the coefficients of noise signal to remove noise. This paper uses the thresholding method with a predetermined threshold used as the evaluation method. All wavelet coefficients less than this threshold are considered as noise and are removed. The rest of the coefficients are considered as clean signals and are kept. 
These clean coefficients later in the final step are reconstructed to obtain an enhanced image.In the next step, the WPTDA is focused on the appropriate wavelet basis selection. The result leads to many choices of best bases. Each base can be considered as an appropriate signal and this makes it important to select the best basis out of the available choices. Several algorithms exist for the best basis selection [9] [10]. In this paper, a kurtosis criterion is used. As stated https://en.wikipedia.org/wiki/Kurtosis\#Interpretation [11], the average value of the standardized data raised to the fourth power. The kurtosis for each node of the wavelet packet tree is estimated and the node with the maximum value is selected as the best basis. The kurtosis is estimated using the (1).

$$
C(y)=\operatorname{cum}(y(n), y(n), y(n), y(n))=M_{i}-3 M_{20}^{2}
$$

where $\mathrm{M}_{\mathrm{i}}$ represents the ith moment about the mean and $\mathrm{y}(\mathrm{n})$ is the vector having the wavelet packet coefficients at each node. Since the fourth order cumulant kurtosis [12] is used to measure the non-Gaussian property of the signal which has a high non-Gaussian distribution. This when combined with the thresholding method exploits the non-Gaussianity in separation module, thus provides a significant gain. The best basis is selected as the node that has the highest kurtosis value.

The next step of WPTDA is thresholding, which consists of two parts. The first part is concerned with the estimation of the threshold value (Step 3) while the second part is to select threshold method (Step 4). A popularly used threshold estimation method is to multiply the coefficients with the median value of the detailed coefficients at each level [13]. In this process, signal energy and standard deviation $(\sigma)$ of every node is used to find the threshold $(\lambda)$. Thus, during each decomposition, the $\sigma$ of the noisy signal is estimated using the (2) given below.

$$
\sigma_{\mathrm{j}}=\operatorname{median}\left(\left|\mathrm{c}_{\mathrm{j}}\right|\right) / 0.6745
$$

where, $\mathrm{j}$ is the decomposition level, $\mathrm{C}_{\mathrm{j}}$ is high frequency wavelet packet coefficients at jth level of decomposition and $\sigma_{\mathrm{j}}$ is called as Median Absolute Deviation (MAD) at this level. The customized threshold value [14] can be gained by the (3).

$$
\lambda=\sigma_{\mathrm{j}} \sqrt{2 \log \left(\mathrm{L}_{\mathrm{j}} \log _{2} \mathrm{~L}_{\mathrm{j}}\right)}
$$

where $L_{j}$ is the length of the noisy signal of jth level. During threshold estimation, it is important to include the detail coefficients at each level as they have positive impact on the robustness of the threshold value. Therefore, the selected threshold has to be rescaled at a particular level. In this research work, the threshold value estimated is dependent on the coefficients at every level. The WPTDA uses a soft thresholding method, which is applied to the high frequency coefficients of the layer. The soft thresholding was used because it reduces abrupt sharp changes and provides an image whose quality is not degraded. Finally, the inverse wavelet packet transformation builds the enhanced tablet image.

\section{B. Independent Component Analysis based Denoising Algorithm}

ICA has been used successfully in blind source signal separation [15] [16] that aim to recover unknown sources from a set of their observations, in which they are mixed in an unknown manner. ICA has the advantage that it can separate hidden source signal from mixed signal in an efficient manner and when combined with image processing, can extract image source signal from noise.

The ICA-based noise removal algorithm consists of the following steps as given in the Fig. 3 .

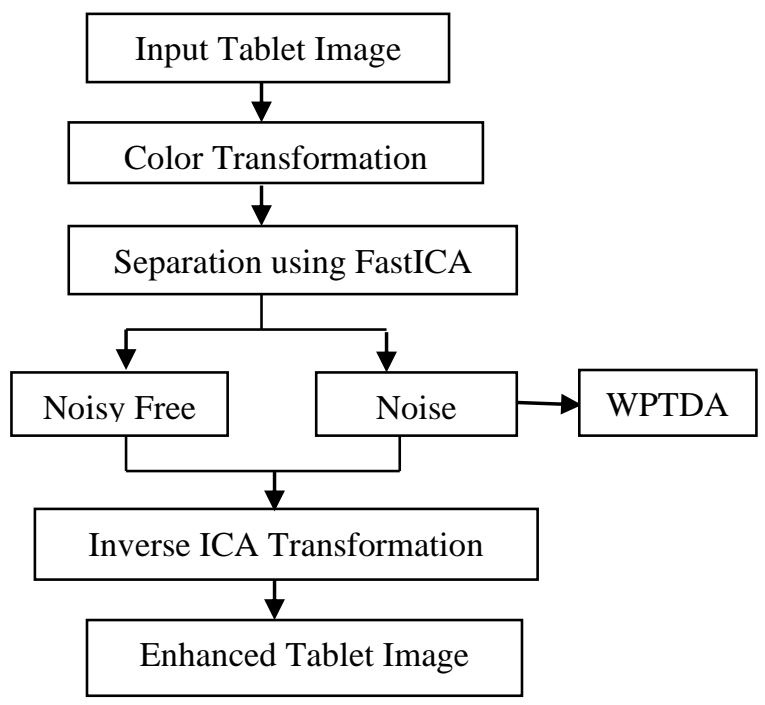

Fig. 3. Steps in ICADA

A basic ICA model can be stated as in (4).

$$
\mathrm{x}(\mathrm{t})=\operatorname{As}(\mathrm{t})
$$

where $\mathrm{x}(\mathrm{t})$ is the observed mixture signal, $\mathrm{A}$ is the unknown mixed matrix, $\mathrm{s}(\mathrm{t})$ is the original source signal, which are assumed to be statistically mutually independent and $n$ is the observed noise signal. Here, $\mathrm{s}$ and $\mathrm{n}$ are considered to be independent to each other. The main goal of ICA is to estimate $\mathrm{s}(\mathrm{t})$ from $\mathrm{x}(\mathrm{t})$, which is equivalent to estimating 'A'. In general, $A$ is estimated using the maximum likelihood method. This ICA model describes the manner in which the observed mixture signal, $\mathrm{x}(\mathrm{t})$, is generated using a process that uses $A$ to mix $s(t)$. This model has the issue while handling noise. Let the noise signal be denoted as $\mathrm{v}(\mathrm{t})$. Then, an ICA model with noise can be denoted as follows (5).

$$
x(t)=A s(t)+v(t)
$$

In the above model, $\mathrm{s}(\mathrm{t})$ (which is an independent component) cannot be directly observed. In case noise is negligible, only the random variables $\mathrm{x}(\mathrm{t})$ are observed and both $\mathrm{s}(\mathrm{t})$ and A must be estimated using $\mathrm{x}(\mathrm{t})$. Now, the ICA solution can be obtained using a learning proces that can find a de-mixing matrix $\mathrm{W}$ or separation inverse matrix of A. Thus, $W$ can be used to yield the independent signals (6).

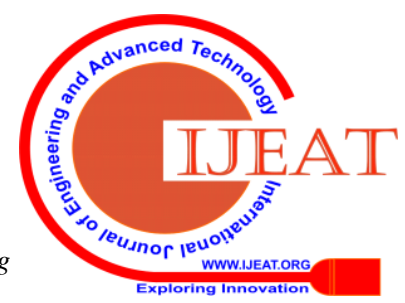




$$
s^{\prime}(t)=w x(t)
$$

where $s^{\prime}(t)$ is the estimation of $s(t)$. When $W=A$, the recovered signal $s^{\prime}(t)$ is exactly the same as $s(t)$. In this scenario, the components of $\mathrm{s}^{\prime}(\mathrm{t})$ are called as ICs (Independent Components).

Several variants of ICA are used for denoising. This paper [17] [5] uses FastICA for removing noise from tablet image. The FastICA algorithm, apart from being the most frequently used algorithm, provides multiple advantages. The algorithm is simple, has fast convergent property and low computational complexity. It is a fixed point iterative algorithm that uses a non-linear function to obtain a global minimum and is applied to the separation matrix $\mathrm{W}$ that is recalculated at each iteration of the algorithm. Once the matrix $\mathrm{W}$ is determined, the independent components can be obtained.

The first step of the FastICA algorithm is whitening (or sphering). This step is a linear transformation that facilitates the separation of the underlying independent signals. Whitening means that the observed vector is linearly transformed to obtain a new vector $\mathrm{X}$ having white. That is, its components are uncorrelated and their variance equals unity. That is, $x(t)$ is made uncorrelated and unit-variance. The whitening is accomplished using Principal Component Analysis (PCA) projection (7).

$$
x^{\prime}(t)=U x(t)
$$

where $x^{\prime}(t)$ is the whitening signal with its data covariance matrix $E\left[x^{\prime}(t) x^{\prime}(t) T\right]$ set to $I, U$ is the whitening matrix, generally computed after singular or eigen value decomposition of the covariance matrix of $\mathrm{x}(\mathrm{t})(8)$.

$$
\mathrm{U}=\Lambda^{-1 / 2} \mathrm{~V}^{\mathrm{T}}
$$

Where $\Lambda=$ diagonal matrix with eigen values.

$\mathrm{E}[\mathrm{x}(\mathrm{t}) \mathrm{x}(\mathrm{t})]$ and $\mathrm{V}=$ matrix with its equivalent eigen vectors.

Now, the ICA model with noise can be obtained by substituting (7) with (8) and is given in (9).

$$
\mathrm{x}^{\prime}(\mathrm{t})=\mathrm{UAs}(\mathrm{t})
$$

And the matrix $\mathrm{B}=\mathrm{UA}$ is orthogonal and thus the solution now takes the following form (10).

$$
s^{\prime}(t)=W x^{\prime}(t)
$$

If $\mathrm{W}=\mathrm{B}_{-1}$, then the recovered signal $\mathrm{s}^{\prime}(\mathrm{t})$ is exact replica of the original signal, $\mathrm{s}(\mathrm{t})$. There are several cost functions that can be used to calculate W. In this paper, the kurtosis is used. Kurtosis can indicate how independent signals are and is a frequently used classical measure of non-Gaussianty.

To summarize, the FastICA method starts with noise free data as training set of the observed signal (input degraded image) and then using some sparse coding method, the orthogonal matrix $\mathrm{W}$ is estimated so that the components of the linearly transformed components have sparse distribution. Next, the algorithm, for each sparse component, estimates a density model. This results with noisy observation of the observed signal. For each of these observations, the shrinkage non-linearity is applied, which results with a set of recovered signal. Finally, an inverse transformation is applied to obtain the denoised image.

\section{COLOR SPACE MODELS}

The color space models help to visualize and specify the color in a more efficient manner. It is defined using a numerical system that describes the various colors in the tablet image and generally consists of three or four numbers to represent a subset of colors representing the primary colors [4] of the tablet images. In image analysis domain, the images are generally represented using RGB color space model [18]. Frequently, this color represented is converted to two other frequently used color models, namely, $\mathrm{YCbCr}$ and HSV color models. All the color spaces define a method of uniquely specifying colors via three numbers. The effect of all these color models on noise removal is analyzed in this paper.

Fig. 4 shows the methodology used while analyzing color space models on denoising. In general, all images are stored using RGB color space model. If in need to use other color space models, a conversion process that converts the color space from RGB to the desired model is required. After conversion, any one of the denoising algorithm (WPTDA / ICADA) is used to remove/reduce the noise. Finally, a reverse color space conversion process is applied to convert the image back to RGB color space format to obtain the final enhanced tablet image.

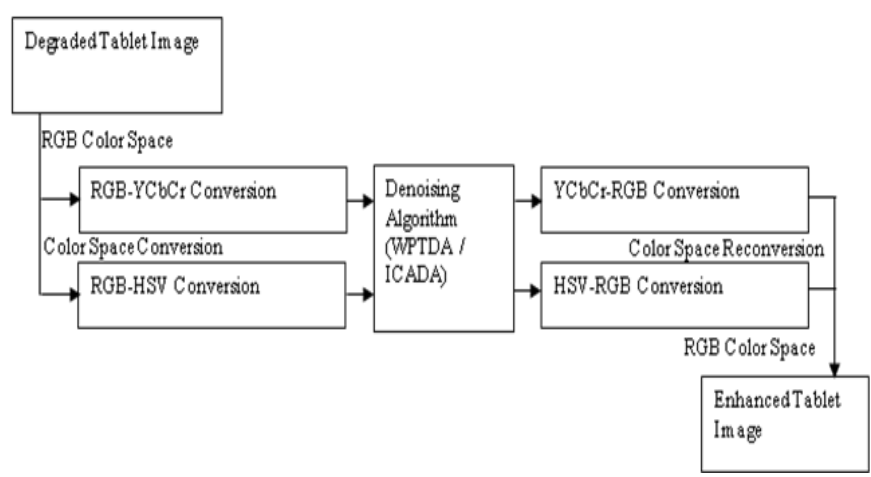

Fig. 4. Analysis of Color Space Models During Denoising

\section{A. RGB to HSV Conversion}

Conversion of RGB color space to HSV is obtained by the $\mathrm{R}^{\prime} \mathrm{G}$ 'B' triplet. Using these values the saturation (S) and Value (V) are calculated using (11) and (12).

$$
\begin{aligned}
& \mathrm{S}=\frac{(\max -\mathrm{min})}{\max } \\
& \mathrm{V}=\max
\end{aligned}
$$

The calculation of $\mathrm{H}$ (Hue) begins by calculating R", G" and B" using (13). 


$$
\mathrm{R}^{\prime \prime}=\frac{\max -\mathrm{R}}{\max -\min } ; \mathrm{G}^{\prime \prime}=\frac{\max -\mathrm{G}}{\max -\min } ; \mathrm{B}^{\prime \prime}=\frac{\max -\mathrm{B}}{\max -\min }
$$

The value of $\mathrm{H}$ is then calculated as follows:

if $\mathrm{S}=0$ then $\mathrm{H}$ is undefined (monochrome image)

else if $\mathrm{R}^{\prime}=\max$

$$
\begin{array}{ll}
\mathrm{G}^{\prime}=\min & \mathrm{H}=5+\mathrm{B}^{\prime \prime} \\
\mathrm{G}^{\prime} \neq \min & \mathrm{H}=1-\mathrm{G}^{\prime \prime}
\end{array}
$$

else if $G^{\prime}=\max$

$$
\begin{array}{ll}
\mathrm{B}^{\prime}=\min & \mathrm{H}=\mathrm{R}^{\prime \prime}+1 \\
\mathrm{~B}^{\prime} \neq \min & \mathrm{H}=3-\mathrm{B}^{\prime \prime} \\
=\max & \mathrm{H}=3+\mathrm{G}^{\prime \prime}
\end{array}
$$

else if $\mathrm{R}^{\prime}=\max$

else $\mathrm{H}=5-\mathrm{R}^{\prime \prime}$

The $\mathrm{H}$ value is then multiplied by 60 degrees, which converts it into degrees giving HSV with $\mathrm{S}$ and $\mathrm{V}$ values between 0 and 1 and $H$ value between 0 and 360 degrees.

The reverse process, which is converting an image in HSV color space to $\mathrm{R}^{\prime} \mathrm{G}^{\prime} \mathrm{B}$ ', first the Hue value in the range 0 to 360 degrees is divided by 60 degrees to obtain the Hex value. From this the primary colors (a, b, and c) are calculated using (14) - (17).

Secondary color $=$ Hex - primary color

$$
\begin{aligned}
& \mathrm{a}=(1-\mathrm{S}) \mathrm{V} \\
& \mathrm{b}=(1-(\mathrm{S} * \text { secondary color })) \mathrm{V} \\
& \mathrm{c}=(1-(\mathrm{S} *(1-\text { secondary color }))) \mathrm{V}
\end{aligned}
$$

From the primary color, the $\mathrm{R}^{\prime} \mathrm{G}^{\prime} \mathrm{B}^{\prime}$ values are then calculated using the following procedure.

$$
\begin{array}{ll}
\text { if primary color }=0 \text { then } & \mathrm{R}^{\prime}=\mathrm{V}, \mathrm{G}^{\prime}=\mathrm{c}, \mathrm{B}^{\prime}=\mathrm{a} \\
\text { else if primary color = } 1 \text { then } & \mathrm{R}^{\prime}=\mathrm{b}, \mathrm{G}^{\prime}=\mathrm{V}, \mathrm{B}^{\prime}=\mathrm{a} \\
\text { else if primary color = 2 then } & \mathrm{R}^{\prime}=\mathrm{a}, \mathrm{G}^{\prime}=\mathrm{V}, \mathrm{B}^{\prime}=\mathrm{c} \\
\text { else if primary color }=3 \text { then } & \mathrm{R}^{\prime}=\mathrm{a}, \mathrm{G}^{\prime}=\mathrm{b}, \mathrm{B}^{\prime}=\mathrm{V} \\
\text { else if primary color }=4 \text { then } & \mathrm{R}^{\prime}=\mathrm{c}, \mathrm{G}^{\prime}=\mathrm{a}, \mathrm{B}^{\prime}=\mathrm{V} \\
\text { else if primary color }=5 \text { then } & \mathrm{R}^{\prime}=\mathrm{V}, \mathrm{G}^{\prime}=\mathrm{a}, \mathrm{B}^{\prime}=\mathrm{b}
\end{array}
$$

\section{B. RGB to YCbCr Conversion}

The conversion process to convert RGB to YCbCr color format and $\mathrm{YCbCr}$ to RGB color format is performed using (18) and (19) respectively.

$$
\left[\begin{array}{c}
\mathrm{Y} \\
\mathrm{Cb} \\
\mathrm{Cr}
\end{array}\right]=\left[\begin{array}{c}
16 \\
128 \\
128
\end{array}\right]+\frac{1}{256}\left[\begin{array}{ccc}
65.738 & 129.057 & 25.064 \\
-37.945 & -74.494 & 112.439 \\
112.439 & -94.154 & -18.285
\end{array}\right]\left[\begin{array}{c}
\mathrm{R} \\
\mathrm{G} \\
\mathrm{B}
\end{array}\right]
$$

$$
\left[\begin{array}{l}
R \\
G \\
B
\end{array}\right]=\frac{1}{256}\left[\begin{array}{ccc}
290.082 & 0 & 408.583 \\
298.082 & -100.291 & -208.120 \\
298.082 & -516.411 & 0
\end{array}\right]\left[\begin{array}{c}
\mathrm{Y}-16 \\
\mathrm{Cb}-128 \\
\mathrm{Cr}-128
\end{array}\right]
$$

\section{EXPERIMENTAL RESULTS}

The performance of the denoising algorithms while varying the color space model used was vigorously tested with several tablet images. The tablet image database was obtained from ftp://ftp.nlm.nih.gov/nlmdata/pir/DC.zip [19] and consists of two types of images, referred to as reference quality and consumer quality images. Reference quality images are high quality images stored in JPEG format. This

\begin{tabular}{|c|c|c|c|c|}
\hline \multirow[b]{2}{*}{$\begin{array}{c}\text { Denoising } \\
\text { Algorithms }\end{array}$} & \multicolumn{4}{|c|}{ Reference Database } \\
\hline & $\begin{array}{c}\text { PSNR } \\
\text { (dB) }\end{array}$ & FoM & MSSI & $\begin{array}{c}\text { Speed } \\
\text { (Seconds) }\end{array}$ \\
\hline WPTDA-E & 39.86 & 0.9416 & 0.8611 & 0.9789 \\
\hline WPTDA-K & 40.11 & 0.9476 & 0.8693 & 0.9724 \\
\hline ICADA & 38.93 & 0.9391 & 0.8397 & 1.0225 \\
\hline FICADA & 39.17 & 0.9453 & 0.8643 & 0.9567 \\
\hline \multirow[b]{2}{*}{$\begin{array}{c}\text { Denoising } \\
\text { Algorithms }\end{array}$} & \multicolumn{4}{|c|}{ Consumer Database } \\
\hline & $\begin{array}{l}\text { PSNR } \\
(d B)\end{array}$ & FoM & MSSI & $\begin{array}{c}\text { Speed } \\
\text { (Seconds) }\end{array}$ \\
\hline WPTDA-E & 38.79 & 0.9115 & 0.8361 & 0.9874 \\
\hline WPTDA-K & 39.63 & 0.9183 & 0.8403 & 0.9813 \\
\hline ICADA & 37.23 & 0.9104 & 0.8389 & 1.0357 \\
\hline FICADA & 37.74 & 0.9162 & 0.8439 & 0.9536 \\
\hline
\end{tabular}
set of images has both their frontal and back view stored. Consumer quality images are images captured using digital camera, as normal public will do and is available at ftp://ftp.nlm.nih.gov/nlmdata/pir/DR.zip [20]. Totally 20 images from the database are used for testing. The result presented in this section is the average values obtained using these 20 images. Performance evaluation analyzed the quality of the denoised image (PSNR), edge preservation capacity of the denoised image (Figure of Merit - FoM), structure maintenance ability (Mean Structural Similarity Index - MSSI) and execution time (CPU Time). The experiments were organized in two stages. Stage 1 experiments evaluated the two algorithms proposed in this paper and compared their performance with its conventional counterparts WPTDA-E and ICADA. Stage 2 experiments analyses the effect of the selected color space models on their capability in producing the enhanced tablet images.

\section{A. Evaluation of Denoising Algorithm}

Table I shows the results obtained by the WPTDA-K (WPTDA using kurkosis for best basis selection) and FICADA (FastICA) algorithms in terms of PSNR, FoM, MSSI and speed. The results are compared with WPTDA using the frequently used entropy best basis selection algorithm (WPTDA-E) and conventional ICA based Denoising Algorithm (ICADA).

Table- I: Evaluation of Denoising Algorithms 
From the results, it is evident that both the algorithms WPTDA-K and FICADA proposed in this paper have improved the denoising capacity than their conventional counterparts WPTDA-E and ICADA with respect to all the selected performance metrics. The conventional ICA based denoising algorithm took maximum time (more than one second) to remove noises from tablet images. Thus, it can be concluded that both WPTDA-K and FICADA are successful to enhance the tablet images. Motivated by these results, the WPTDA-K and FIADA algorithms are used in the analysis of the selected color space models.

\section{B. Comparison of Color Space Models}

The coding scheme used during discussion is given in Table II.

Table- II: Coding Scheme Used

\begin{tabular}{|l|l|}
\hline \multicolumn{1}{|c|}{ Algorithm } & \multicolumn{1}{|c|}{ Code } \\
\hline WPTDA-K with RGB Color Space Mode & W-R \\
\hline WPTDA-K with YCbCr Color Space Mode & W-Y \\
\hline WPTDA-K with HSV Color Space Mode & W-H \\
\hline FICADA with RGB Color Space Mode & I-R \\
\hline FICADA with YCbCr Color Space Mode & I-Y \\
\hline FICADA with HSV Color Space Mode & I-H \\
\hline
\end{tabular}

Fig. 5a and 5b shows the average PSNR values obtained by the WPTDA and ICADA algorithms respectively while varying the color space model used and when tested using reference and consumer databases.

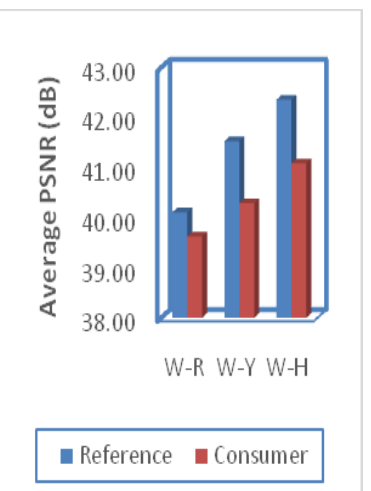
Algorithms (a) WP-Based

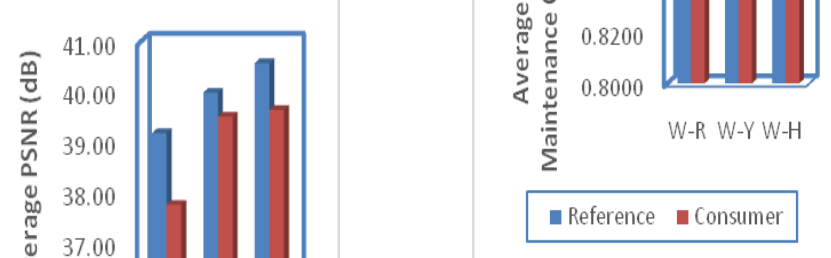

(a) WP-Based Algorithms algorithms shows that the WPTDA algorithm works better at removing noise than FICADA algorithm.

Fig. $6 \mathrm{a}$ and $6 \mathrm{~b}$ shows the edge preservation capacity of the algorithms after denoising with reference and consumer quality image databases, respectively. The structure preservation capacity of the algorithms after denoising with reference and consumer quality image databases respectively is shown in Fig. 7a and 7b.

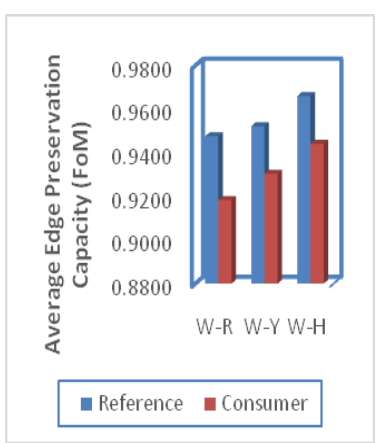

(a) WP-Based Algorithms

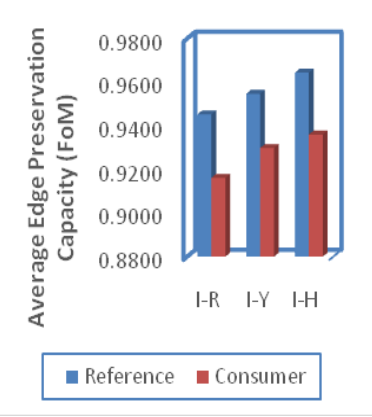

(b) FastICA-Based Algorithms
Fig. 6. Edge Preservation Capacity (FoM)Analysis

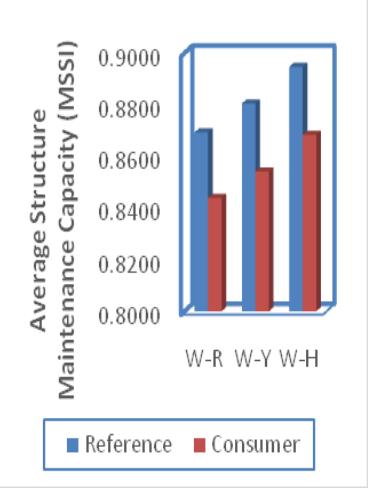

Fig. 7. Structure Preservation Capacity (MSSI) Analysis

The results obtained again stress the fact that the usage of HSV color space model preserves edges in the enhanced image in a better manner when compared with RGB and YCbCr color space models. This is clear from the high FoM values obtained by $\mathrm{W}-\mathrm{H}$ and $\mathrm{I}-\mathrm{H}$ models. Similarly, the denoising algorithms with HSV color space also has better structure preserving capacity, as evident from the near to unity MSSI values obtained by W-H and I-H algorithms. This trend obtained was the same with both the reference and consumer databases.The speed of the denoising algorithms (in terms of CPU time) with different color space models (see Table III).

Table- III: Analysis of Average Speed (CPU Time) in Seconds that with respect to the quality of the denoised image in terms of PSNR, the WPTDA-K algorithm using HSV color space model produces the maximum efficiency.

The WPTDA-K algorithm showed an average efficiency gain of $2.34 \%$ (Reference) and $4.76 \%$ (Consumer) with respect to PSNR, 0.24\% (Reference and Consumer) with respect to FoM and $0.58 \%$ (Reference) and $0.16 \%$ (Consumer) with respect to MSSI performance metrics. Thus, the comparison of the WPTDA-K and FICADA 


\begin{tabular}{|c|c|c|}
\hline W-H & 0.9736 & 0.9803 \\
\hline I-R & 0.9567 & 0.9536 \\
\hline I-Y & 0.9573 & 0.9625 \\
\hline I-H & 0.9571 & 0.9624 \\
\hline
\end{tabular}

Examining the speed of the denoising algorithm is diverse from the other performance metrics. Here, the denoising algorithm based on FastICA is the fastest algorithm to produce the enhanced image and the algorithm using RGB color space model produces quick results. However, as quality, edges and structure have more importance during tablet identification, it can concluded that the WPTDA and FICADA algorithms with HSV color space model as the algorithm that produces maximum benefits.

Fig. 8 shows the effect of using these algorithms on tablet identification. For this purpose, the system proposed by [13] was used. This system is referred to as Supervised Descent Method (SDM). The accuracy performance metric was used for this purpose.

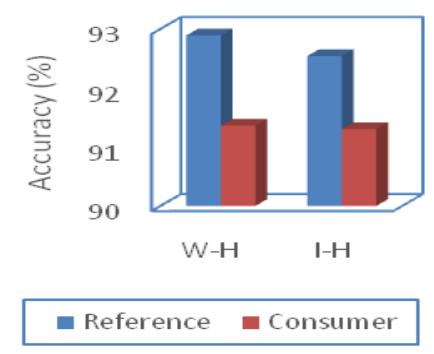

Fig. 8. Accuracy (\%) Analysis

\section{Visual Results}

One sample reference image along with its consumer counterpart was randomly selected for showing the visual results as shown in Fig. 9. The denoised outputs of these tablets are shown in Fig. 10.

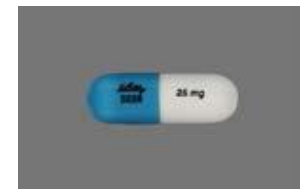

(a) Reference Image 1

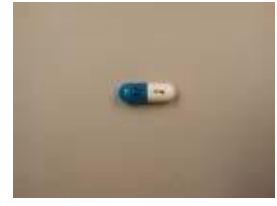

(b) Consumer Image of (a)
Fig. 9. Sample Reference and its Consumer Tablet Images

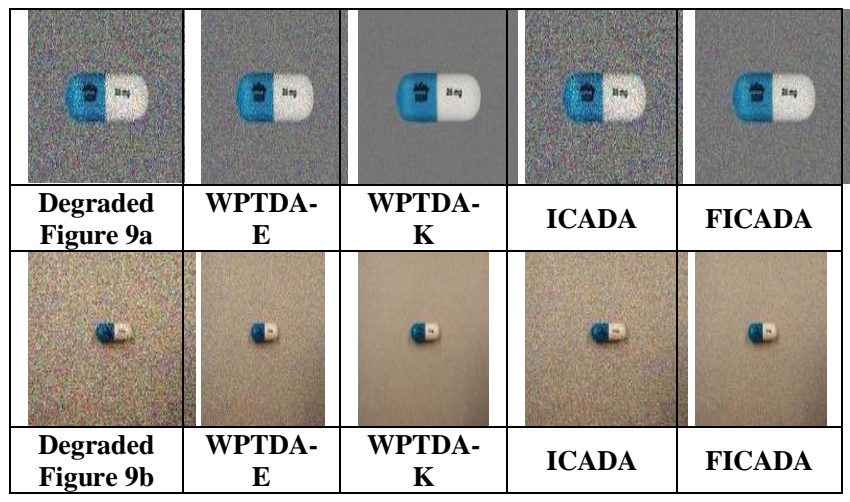

\section{CONCLUSION AND FUTURE WORK}

This paper presented two denoising algorithms based on wavelet packets and independent component analysis to remove noise present in tablet images. The wavelet packet denoising algorithm was enhanced through the use of kurtosis method to select best basis, while Fast ICA, which is an advanced version of conventional ICA was used. Further, the paper also compared the effect of three different color space model (RGB, YCbCr and HSV) on denoising. The performance is analyzed using parameters like PSNR, FoM, MSSI and Speed. Two stages of experiments were conducted to remove noise and to analyze the color space models. Experimental results showed that the HSV color space model produced maximum efficiency during denoising with both wavelet packet and Fast ICA algorithms. The experiments also showed that the wavelet packet based algorithm is better with preserving the quality of the tablet images, while the ICA based algorithm has the advantage being fast at removing noise. Future research will focus on designing an algorithm that can combine these advantages. Further, other preprocessing steps like illumination and lighting variations, contrast adjustment, edge enhancement and normalization are also to be probed.

\section{REFERENCES}

1. D. Faggella, "Machine learning healthcare applications - 2018 and Beyond, Tech emergence", (2018, August). [Online]. Available: https://www.techemergence.com/machine-learning-healthcare application

2. IEEE Technical Committee, "Automation in Health Care Management, IEEE Robotics \& Automation Society”, (2018, August). [Online]. Available: $\quad$ http://www.ieee-ras.org/automation-in-health-caremanagement

3. A.B. Dhivya and M. Sundaresan, "Performance analysis of interpolation methods for improving sub-image content-based retrieval", Proceedings of the INDIACom 3rd International Conference on Computing for Sustainable Global Development, 2016, pp. 1-5.

4. P. Nalini, and B.L. Malleswari, "Performances of Different Color Representations in Image Retrieval and Classification: A Comparative Analysis", International Journal of Emerging Engineering Research and Technology, Vol. 4, Issue 11, 2016, pp. 12-18.

5. M.R. Mirarab, H. Dehghani, and A. Pourmohammad, "A novel wavelet based ICA technique using Kurtosis”, 2nd International Conference on Signal Processing Systems, 2010, pp. V1-36-V1-39.

6. Joblove, H. George, and Donald Greenberg, "Color spaces for computer graphics", ACM sig graph computer graphics, Vol. 12, No. 3, 1978.

7. M. Kimlyk, and S. Umnyashkin, "Image denoising using discrete wavelet transform and edge information", IEEE Conference of Russian Young Researchers in Electrical and Electronic Engineering, 2018, pp. 1823-1825.

8. R. Haddadi, E. Abdelmounim and A. Belaguid, "Discrete Wavelet Transform Based Algorithm for Recognition of QRS Complexes", World of Computer Science and Information Technology, Vol. 4, No. 9, 2014, pp. 127-132.

9. A. Cardinali, and G.P. Nason, "Locally Stationary Wavelet Packet Processes: Basis Selection and Model Fitting", Journal of Time Series Analysis, Special Issue, John Wiley \& Sons Ltd., Vol. 38, Issue 2, 2017, pp. 151-174.

10. J. Galka and M. Zilko, "Best Basis selection of the Wavelet Packet Cosine Transform in speech analysis", AFRICON, 2009, pp.1-4.

11. (2018, August). [Online]. Available: https://en.wikipedia.org/wiki/Kurtosis\#Interpretation

12. E. Oja, A. Hyvarinen and J. Karhunen, "Independent Component Analysis", John Wiley \& Sons, Inc, 2001.

13. R. Sharma and V.P. Pyara, "A Robust Denoising Algorithm for Sounds of Musical Instruments Using Wavelet Packet Transform”, Circuits and Systems, Vol. 4, No. 7, 2013, pp. 459-465.

Fig. 10. Visual Results of Denoising Algorithms 
14. M.T. Johnson, X. Yuan and Y. Ren, "Speech Signal Enhancement through Adaptive Wavelet Thresholding”, Speech Communication, Vol. 49, No. 2, 2007, pp. 123-133.

15. H. Sawada, S. Araki, R. Mukai, and S. Makino, "Blind extraction of dominant target sources using ICA and time-frequency masking”, IEEE Transactions on Audio, Speech and Language Processing, Vol. 14, No. 6, 2006, pp. 2165-2173.

16. T. Kim, H.T. Attias, S.Y. Lee and T.W. Lee, "Blind source separation exploiting higher-order frequency dependencies", IEEE Transactions on Audio, Speech and Language Processing,Vol. 15, No. 1, 2007, pp. 70-79.

17. M.Y. Abbass, S.A. Shehata, S.S. Haggag, S.M. Diab, B.M. Salam, S El-Rabaie and F.E. El-Samie, "Blind Source Separation with Wavelet Based ICA Technique using Kurtosis", International Conference on Computer Theory and Applications, Vol. 5, No. 9, 2013, pp. 417-421.

18. (2018, August). [Online]. http://en.wikipedia.org/wiki/RGB_color_model

19. [Online]. Available: ftp://ftp.nlm.nih.gov/nlmdata/pir/DC.zip

20. [Online]. Available: ftp://ftp.nlm.nih.gov/nlmdata/pir/DR.zip

\section{AUTHORS PROFILE}

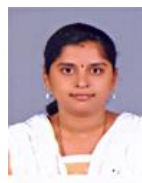

A.B. Dhivya, completed her B.Sc [Computer Science] in Maharaja Arts and Science College, Coimbatore during the year 2002 - 2005 and MCA degree in the same college during 2005 - 2008. She has completed her M.Phil degree in Avinashilingam University for Women, Coimbatore during the period 2009- 2010. She is currently pursuing her Ph.D degree in Computer Science in Bharathiar University, Coimbatore. She got distinction in her PG degree. She received best poster award in 106th Indian Science Congress held at Lovely Professional University, Phagwara, Jalandhar during 2018. Her area of interest is image processing, data mining, and pattern recognition. She attended national workshops and seminars. She also attended and presented papers in national and international conferences. She got fellowship in bharathiar university sponsored by DST PURSE. She is a life member in Indian Science Congress Association and Computer Society of India.

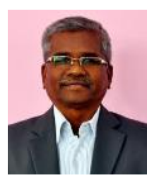

M.Sundaresan is working as Professor and Head of the Department of Information Technology at Bharathiar University, Coimbatore, India. He holds Ph.D in Computer Science. He has contributed more than 50 research papers in different areas of Computer Science and Information Technology such as Image Processing, Data Compression, Natural Language Processing, Speech Processing and Cloud Computing in reputed journals. He is a Senior Life member of Professional bodies such as Computer Society of India, Indian Science Congress Association, Indian Society for Technical Education and IACSIT. He has been an invited keynote speaker for many international conferences. He has also presented research papers in many international conferences/ seminars/ workshops. He has received research grants from various funding agencies. He is also in editorial board of five journals. He was the Sectional President for Information and Communications Science \& Technology (including Computer Sciences) section in 105th Indian Science Congress during 2018. He is the Regional Vice President for Region VII of Computer Society of India. So far he has authored 4 book chapters for 4 edited books (2 for IGI Global USA). 